EPJ manuscript No.

(will be inserted by the editor)

\title{
Near threshold production of the pseudoscalar mesons at the COSY-11 facility
}

\author{
P. Moskal ${ }^{1,2}$, R. Czyżykiewicz ${ }^{1,2}$, H.-H. Adam ${ }^{3}$, A. Budzanowski ${ }^{4}$, E. Czerwiński ${ }^{1}$, D. Gil ${ }^{1}$, D. Grzonka ${ }^{2}$, M. Janusz ${ }^{1,2}$, \\ L. Jarczyk ${ }^{1}$, B. Kamys ${ }^{1}$, A. Khoukaz ${ }^{3}$, K. Kilian ${ }^{2}$, P. Klaja ${ }^{1,2}$, W. Oelert ${ }^{2}$, C. Piskor-Ignatowicz ${ }^{1}$, J. Przerwa ${ }^{1,2}$, \\ B. Rejdych ${ }^{1}$, J. Ritman ${ }^{2}$, T. Sefzick ${ }^{2}$, M. Siemaszko ${ }^{5}$, M. Silarski $^{1}$, J. Smyrski ${ }^{1}$, A. Täschner ${ }^{3}$, M. Wolke ${ }^{2}$, P. Winter ${ }^{6}$, \\ P. Wüstner ${ }^{2}$, M. J. Zieliński ${ }^{1}$, W. Zipper ${ }^{5}$ \\ 1 Institute of Physics, Jagellonian University, 30-059 Cracow, Poland \\ 2 IKP \& ZEL, Forschungszentrum Jülich, 52425 Jülich, Germany \\ 3 IKP, Westfälische Wilhelms-Universität, 48149 Münster, Germany \\ 4 Institute of Nuclear Physics, 31-342 Cracow, Poland \\ 5 Institute of Physics, University of Silesia, Katowice, Poland \\ 6 Department of Physics, University of Illinois at Urbana-Champaign, Urbana, IL 61801, USA
}

Received: date / Revised version: date

\begin{abstract}
We summarise measurements of the COSY-11 collaboration concerning the excitation functions of the near threshold pseudoscalar meson production in the proton-proton interaction. The results are discussed in the context of the meson-nucleon and hyperon-nucleon interactions. We conclude that the $\eta$-proton interaction is significantly stronger than the $\eta^{\prime}$-proton interaction. Similarly, we found that the hyperon $\Lambda$ interacts with the nucleon considerably stronger than the hyperon $\Sigma$, and that the interaction of $K^{-}$-proton is much stronger than this of the $\mathrm{K}^{+}$-proton.
\end{abstract}

PACS. 14.40.-n Mesons - 14.40.Aq $\pi$, K, and $\eta$ mesons - 13.60.Le Meson production

\section{Introduction}

In the low energy regime of the quantum chromodynamics, where the interaction between quarks and gluons cannot be treated perturbatively, hadrons become the relevant degrees of freedom and the knowledge of their interactions is of the basic importance. In this article we will present results of the investigations of the mesonnucleon and hyperon-nucleon interactions based on the shape of the near threshold total cross section excitation functions for the production of the hyperons and pseudoscalar mesons. Due to the space limitation we will restrict the discussion to the conclusions derived from the total cross sections of the proton-proton collisions only.

\section{$2 \mathrm{~K}^{+} \mathrm{K}^{-}$and $\mathrm{K}$-proton interaction}

An important feature of near-threshold measurements is connected with the fact that due to the proximity of bound or quasi-bound states of some of the reaction products, interaction between them can be very strong, thus influencing the measured cross sections essentially. This creates an opportunity to investigate interaction between particles which cannot be accessed in direct elastic scattering experiments. For example, measurements of the reaction $p p \rightarrow p p K^{+} K^{-}$allow one to investigate the kaon- antikaon interaction. Such measurements can help us to understand the nature of the scalars $f_{0}(980)$ and $a_{0}(980)$ which masses are very close to the mass of a kaon pair. The nature of these objects remains a long-standing problem of the meson physics. The standard quark model has difficulties with interpreting these mesons as quark-antiquark pair. Therefore, various non $q-\bar{q}$ descriptions have been proposed including a four-quark system [1], or a kaonantikaon molecule [2,3,4]. Especially for the formation of the molecule, the strength of the kaon-antikaon interaction is of the crucial importance. For study of this interaction we have carried out measurements of the $p p \rightarrow p p K^{+} K^{-}$ reaction near the kinematical threshold. The measurements were conducted using the COSY-11 apparatus [5, 6,7 and the cooler synchrotron COSY 8. They were based on kinematically complete reconstruction of positively charged ejectiles while the negative kaon was identified via the missing mass $9,10,11$. Our results combined with the data obtained by DISTO 14 and ANKE 12,13. collaborations are clearly showing that towards the lower values of the excess energy $Q$ the total cross section value is exceeding expectations based on the homogeneous phase space population including the $p p$ final state interaction (FSI), as it can be seen in Figure 1 The observed difference might originate from the $p K$ and/or $K K$ FSI. We investigated the effect of the interaction between particles in 


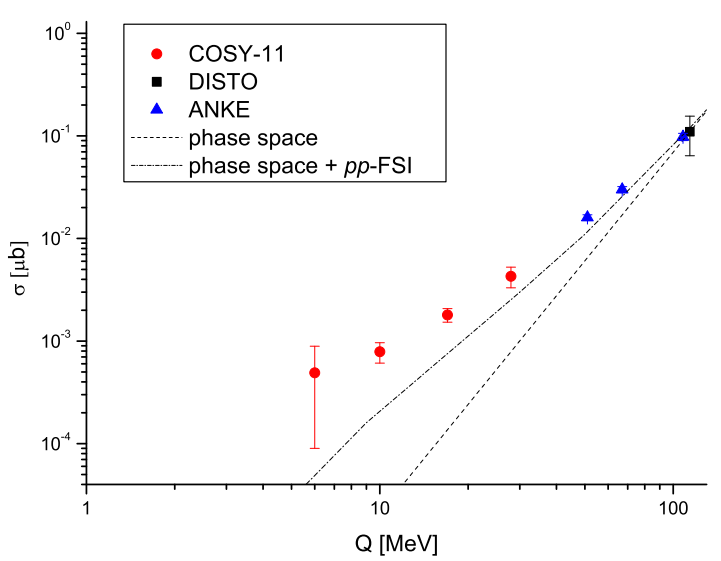

Fig. 1. Total cross section for the reaction $p p \rightarrow p p K^{+} K^{-}$, 10,11, 12, 13, 14 as a function of the excess energy $Q$. The COSY-11 data points 9, 10,11, lie significantly above the expectations indicated by the lines that are normalized to the data point measured by the DISTO collaboration [14].

the final state using also distributions of invariant masses of $p K^{+}, p K^{-}$and $K^{+} K^{-}$pairs [11. Within the limited statistics the distribution for the $p K^{-}$pairs shows an enhancement towards lower masses. Moreover, the enhancement remains when the distribution of the $p K^{-}$invariant mass is normalized to the corresponding $p K^{+}$spectrum, indicating that the $p K^{-}$interacts much stronger than the $p K^{+}$[11,40. Indeed, in the recent article of the ANKE collaboration it is shown that most of the closeto-threshold enhancement of the excitation function can be explained by the $K^{-}$-proton interaction. However, the data points closest to the threshold are still above this predictions indicating the signal from the KK or $\mathrm{K}^{+}$-proton interaction. For a strict description of the final state, calculations based on application of the four-body formalism are required.

\section{Hyperon-nucleon interaction}

The existence of light hypernuclei, such as ${ }^{3} \mathrm{He}_{\Lambda}$, shows the low energy $\Lambda$-p interaction to be strongly attractive, though not sufficient to bind the hyperon-deuteron 22 . The hyperon-nucleon interaction is of special interest since it is influenced by the strange quark content of the hyperon. However, in contrast to the nucleon- nucleon case, due to the short lifetime of hyperons, the direct measurements of low-energy hyperon-nucleon scattering are sparse and the resulting parameters are rather poorly known 22 . Using the COSY-11 facility we have determined the excitation function of the $p p \rightarrow p K^{+} \Lambda, p p \rightarrow p K^{+} \Sigma^{0}$ and $p p \rightarrow n K^{+} \Sigma^{+}$reactions in the near threshold energy range. Surprisingly, the total cross section for the production of the hyperon $\Lambda$ was found to be by a factor of thirty larger than this for $\Sigma^{0}$. It is in drastic contrast to the results of the cross section ratio $\sigma(p p \rightarrow$ $\left.p K^{+} \Lambda\right) / \sigma\left(p p \rightarrow p K^{+} \Sigma^{0}\right)$ determined at higher energies, where it was found to be equal to three as expected from the isospin relations. This observation raised an interesting question whether the drastic increase of the cross section ratio near threshold is a mere effect of the $\Lambda$-p interaction or whether it is due to the reaction mechanism. To explain this unexpected increase different models have been proposed based on the coherent exchange of the $\pi$ and K mesons 23 or on the excitation of the intermediating resonances [24, 25, 26, 27. All these models failed however to predict the value of the total cross section for the $p p \rightarrow$ $n K^{+} \Sigma^{+}$reaction. To understand the hyperon-nucleon interaction a further thorough theoretical investigations are needed. Figure 2 presents the COSY-11 data together

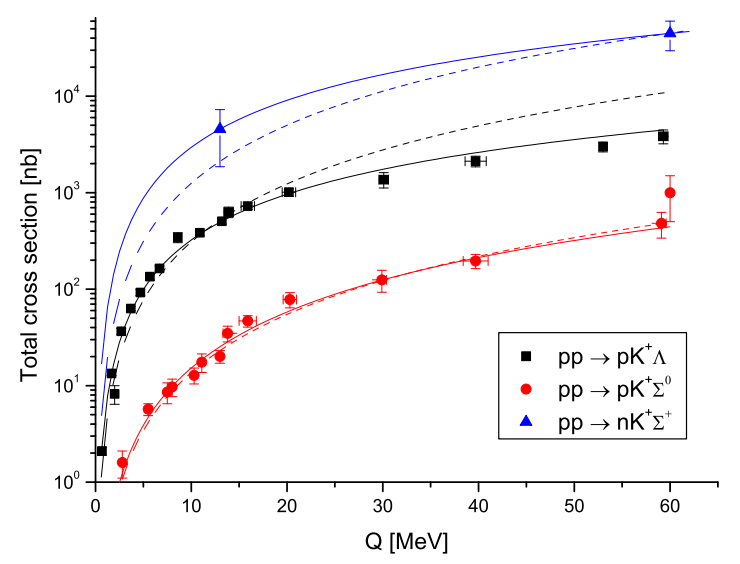

Fig. 2. Total cross section as function of the excess energy $Q$ for the near threshold production of the hyperons $\Lambda, \Sigma^{0}$ and $\Sigma^{+}$via the $p p \rightarrow p K^{+} \Lambda$ 15, 16, 17, 18, 19, $p p \rightarrow p K^{+} \Sigma^{0}$ [16 17, 18, 20, and $p p \rightarrow n K^{+} \Sigma^{+}$[21] reactions, respectively.

with expectations derived under the assumption of the homogeneously populated phase space (dashed lines) and the phase-space modified by the hyperon-nucleon interactions (solid lines). The comparison of the calculations with the data suggests much weaker final-state interaction in the $\mathrm{p}-\Sigma^{0}$ channel than in the case of the $\mathrm{p}-\Lambda$. Therefore, the most plausible seems to be an explanation that the observed enhancement of the production of the $\Lambda$ over the $\Sigma$ hyperon is predominantly due to the large $\Lambda$-proton interaction and relatively negligible $\Sigma$-proton interaction. This would also explain why the $\Lambda$-hypernuclei are observed and there are no $\Sigma$-hypernuclei [40]

Regarding the n- $\Sigma^{+}$interaction due to the large systematic uncertainties, and only two available data points, any conclusions would be at present premature.

\section{Interaction of the $\eta$ and $\eta^{\prime}$ mesons with protons}

In this section we give account of the studies of the interactions between the $\eta$ and $\eta^{\prime}$ mesons with nucleons. It is rather challenging to conduct such research because 
these mesons decay within a distance of tens of femtometers rendering their direct detection impossible. It is also completely unfeasible to accomplish out of them a beam or a target. Therefore, we have produced these mesons in the collisions of protons close to the kinematical threshold where the outgoing particles possess low relative velocities and remain in the distance of few femtometers long enough to experience the strong interactions which may manifests itself in a measurable manner.

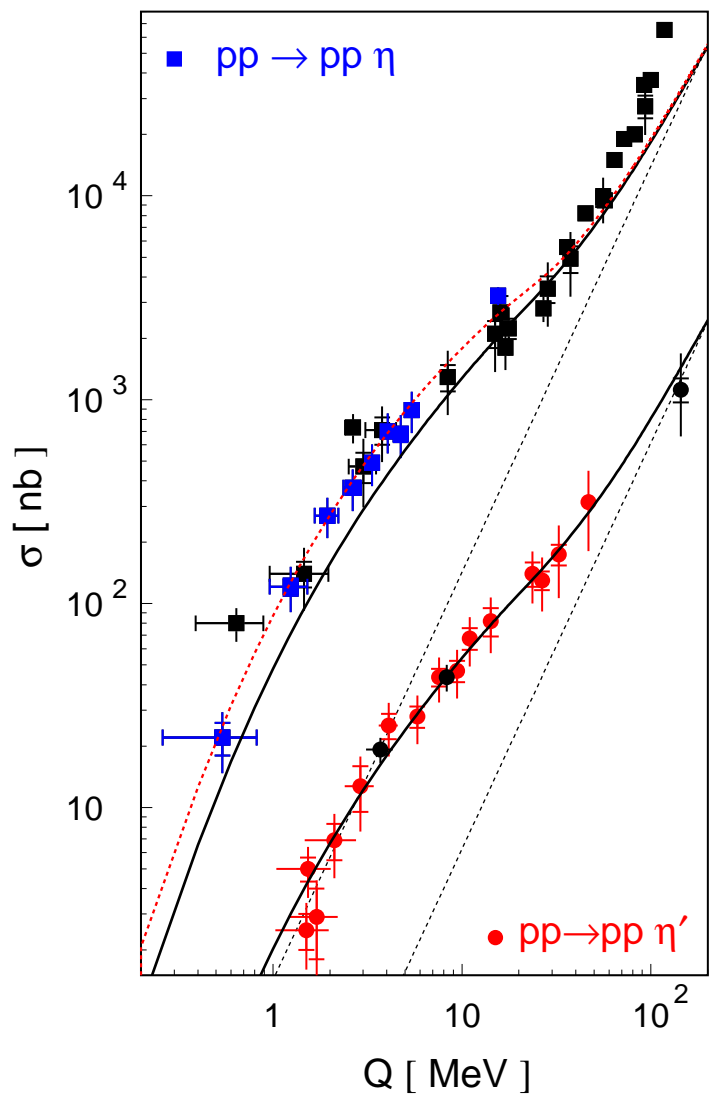

Fig. 3. Total cross section as a function of the excess energy $\mathrm{Q}$ for the reactions $p p \rightarrow p p \eta$ 28, 29, 30,31,32,33,34 (squares) and $p p \rightarrow p p \eta^{\prime}$ 35, 36, 37, 38, 39. (circles). The results determined using the COSY-11 facility and the synchrotron COSY (red and blue) are shown together with the data from the CELSIUS and SATURNE facilities (black). The dashed lines indicate a phase space integral normalized arbitrarily. The solid lines show the homogeneous phase space ditribution with inclusion of the proton-proton strong and Coulomb interaction. Results of calculations taking into account additionally the interaction between the $\eta$ meson and the proton is presented by the red dotted line.

Measurements of the $p p \rightarrow p p \eta$ and $p p \rightarrow p p \eta^{\prime}$ reactions have been conducted close to the kinematical threshold. The obtained near threshold excitation functions are presented in Figure 3. The remarkable difference between the shape of the excitation function of the $p p \rightarrow p p \eta$ and $p p \rightarrow p p \eta^{\prime}$ reactions allowed to conclude that the interaction between the $\eta^{\prime}$ meson and the proton is significantly weaker than the analogous $\eta$-proton interaction [41,42.
This is the first ever empirical appraisal of this hitherto entirely unknown force. Using the COSY-11 facility we have also determined two-particle invariant mass distributions for the production of both the $\eta$ and the $\eta^{\prime}$ meson. The data were taken at the same excess energy in order to enable the direct comparison of the experimental distributions [41. The final results will be reported elsewhere in the near future.

\section{Acknowledgment}

We acknowledge the support of the European CommunityResearch Infrastructure Activity under the FP6 programme (Hadron Physics, RII3-CT-2004-506078), the support of the Polish Ministry of Science and Higher Education under the grants No. 3240/H03/2006/31 and 1202/DFG/2007/03, and the support of the German Research Foundation (DFG).

\section{References}

1. R. L. Jaffe, Phys. Rev. D 15 (1977) 267.

2. C. Hanhart, Eur. Phys. J. A31 (2007) 543.

3. J. D. Weinstein, N. Isgur, Phys. Rev. D 41 (1991) 2236.

4. D. Lohse et al., Nucl. Phys. A 516 (1990) 513.

5. S. Brauksiepe et al., Nucl. Instr. \& Meth. A 376 (1996) 397.

6. J. Smyrski et al., Nucl. Instr. and Meth. A 541 (2005) 574.

7. P. Klaja et al., AIP Conf. Proc. 796 (2005) 160.

8. D. Prasuhn et al., Nucl. Instr. \& Meth. A 441 (2000) 167.

9. M. Wolke, PhD thesis, Univesity of Münster (1997).

10. C. Quentmeier et al., Phys. Lett. B 515 (2001) 276.

11. P. Winter et al., Phys. Lett. B 635 (2006) 23.

12. Y. Maeda et al., e-Print: arXiv:0710.1755 [nucl-ex] (2007).

13. I. Keshelashvili, PhD thesis, University of Tbilisi (2006).

14. F. Balestra et al., Phys. Rev. C 63 (2001) 024004.

15. J.T. Balewski et al., Phys. Lett. B 420 (1998) 211.

16. S. Sewerin et al., Phys. Rev. Lett. 83 (1999) 682.

17. P. Kowina et al., Eur. Phys. J. A 22 (2004) 293.

18. R. Bilger et al., Phys. Lett. 420 (1998) 217.

19. S. Abd El-Samad et al., Phys. Lett. B 632 (2006) 27.

20. M. Fritsch, PhD thesis, University of Erlangen (2002).

21. T. Rożek et al., Phys. Lett. B 643 (2006) 251.

22. J. T. Balewski et al., Eur. Phys. J A 2 (1998) 99.

23. A. Gasparian et al., Nucl. Phys. A 684 (2001) 397.

24. K. Tsushima et al., Phys. rev. C 59 (1999) 369.

25. A. Sibirtsev et al., Nucl. Phys. A 646 (1999) 427.

26. R. Shyam et al., Phys. Rev. C 63 (2001) 022202.

27. R. Shyam, Phys. Rev. C 73 (2006) 035211.

28. F. Hibou et al., Phys. Lett. B 438 (1998) 41;

29. E. Chiavassa et al., Phys. Lett. B 322 (1994) 270;

30. H. Calén et al., Phys. Lett. B 366 (1996) 39;

31. A. M. Bergdolt et al., Phys. Rev. D 48 (1993) 2969;

32. H. Calén et al., Phys. Rev. Lett. 79 (1997) 2642;

33. J. Smyrski et al., Phys. Lett. B 474 (2000) 182;

34. P. Moskal et al., Phys. Rev. C 69 (2004) 025203;

35. F. Balestra et al., Phys. Lett. B 491 (2000) 29.

36. R. Wurzinger et al., Phys. Lett. B 374 (1996) 283.

37. P. Moskal et al., Phys. Lett. B 474 (2000) 416.

38. P. Moskal et al., Phys. Rev. Lett. 80 (1998) 3202.

39. A. Khoukaz et al., Eur. Phys. J. A 20 (2004) 345.

40. C. Wilkin, e-Print: arXiv:0709.0286 [nucl-ex].

41. P. Moskal, e-Print Archive: hep-ph/0408162.

42. P. Moskal et al., Phys. Lett. B 482 (2000) 356. 